\title{
Short Intracortical and Surround Inhibition Are Selectively Reduced during Movement Initiation in Focal Hand Dystonia
}

\author{
Sandra Beck, ${ }^{1,2}$ Sarah Pirio Richardson, ${ }^{1,3}$ Ejaz A. Shamim, ${ }^{1}$ Nguyet Dang, ${ }^{1}$ Martin Schubert, ${ }^{4}$ and Mark Hallett ${ }^{1}$ \\ ${ }^{1}$ Human Motor Control Section, National Institute of Neurological Disorders and Stroke, National Institutes of Health, Bethesda, Maryland 20892-1428, \\ ${ }^{2}$ Department of Clinical Neurology and Neurophysiology, University of Freiburg, D-70196 Freiburg, Germany, ${ }^{3}$ Department of Neurology, University of \\ New Mexico, Albuquerque, New Mexico 87106, and ${ }^{4}$ Spinal Cord Injury Centre, University Hospital Balgrist, 8008 Zurich, Switzerland
}

\begin{abstract}
In patients with focal hand dystonia (FHD), pathological overflow activation occurs in muscles not involved in the movement. Surround inhibition is a neural mechanism that can sharpen desired movement by inhibiting unwanted movement in adjacent muscles. To further establish the phenomenon of surround inhibition and to determine whether short intracortical inhibition (SICI) reflecting inhibition from the local interneurons in primary motor cortex (M1), might play a role in its genesis, single- and paired-pulse transcranial magnetic stimulation (TMS), and Hoffmann reflex testing were applied to evaluate the excitability of the relaxed abductor pollicis brevis muscle (APB) at various intervals during a movement of the index finger in 16 patients with FHD and 20 controls. Whereas controls showed inhibition of APB motor-evoked potential (MEP) size during movement initiation and facilitation of APB MEP size during the maintenance phase, FHD patients did not modulate APB MEP size. In contrast, SICI remained constant in controls, but FHD patients showed reduced SICI during movement initiation. The $H_{\max } / M_{\max }$ ratio in control subjects increased during movement initiation. The results provide additional evidence for the presence of surround inhibition in M1, where it occurs only during movement initiation, indicating that different mechanisms underlie movement initiation and maintenance. Thus, surround inhibition is sculpted both in time and space and may be an important neural mechanism during movement initiation to counteract increased spinal excitability. SICI may contribute to its generation, because in patients with FHD, the lack of depression of APB MEP size is accompanied by a reduction in SICI.
\end{abstract}

Key words: dystonia; motor control; motor cortex; inhibition; human; coordination

\section{Introduction}

A basic issue in the neurophysiology of motor control is how the brain generates the complex spatiotemporal commands needed to vary speed, amplitude, and direction of finger movements (Wing, 2002; Doumas et al., 2008). This skill is impaired in patients with focal hand dystonia (FHD), such as writer's cramp or musician's cramp (Chen et al., 1998). One physiological mechanism to focus neuronal activity and to select neuronal responses is the suppression of excitability in an area surrounding an activated neural network. This mechanism is called surround inhibition and was first described in the retina, where cells are excited by light that falls in the center of their receptive field, whereas light in the periphery has an inhibitory effect on the same cell (Angelucci et al., 2002). In the sensory system, surround inhibi-

Received July 29, 2008; revised Aug. 27, 2008; accepted Aug. 28, 2008.

This work was supported by the Deutsche Forschungsgemeinschaft (BE-3792/1) and the Intramural Research Program of the National Institutes of Health-National Institute of Neurological Disorders and Stroke. We thank D. Schoenberg for skillful editing.

Correspondence should be addressed to Dr. Sandra Beck, Human Motor Control Section, National Institute of Neurological Disorders and Stroke, National Institutes of Health, Building 10/5N240, 10 Center Drive, Bethesda, MD 20892-1428. E-mail:becksa@ninds.nih.gov.

DOI:10.1523/JNEUROSCI.3564-08.2008

Copyright $\odot 2008$ Society for Neuroscience $\quad$ 0270-6474/08/2810363-07\$15.00/0 tion provides spatiotemporal discrimination of various sensory inputs (Blakemore et al., 1970).

The notion that surround inhibition may be a relevant mechanism in the motor system and could aid the selective execution of desired movements in humans goes back at least to the work of Denny-Brown (1967), and more recently, an anatomical hypothesis was proposed by Mink (1996). Meanwhile, there are several studies supporting the presence of surround inhibition ( $\mathrm{Zi}$ emann et al., 1996; Hallett, 2003, 2004; Sohn and Hallett, 2004b), although its mechanism both physiologically and anatomically is still to be determined. Transcranial magnetic stimulation (TMS) allows motor cortical excitability and corticocortical inhibitory circuits to be assessed noninvasively. During motor activation, active muscles show increased excitability while neighboring, nonsynergistic muscles are inhibited (Sohn and Hallett, 2004b; Shin et al., 2007). We consider this effect to be a demonstration of surround inhibition in the motor system.

However, it is unclear, how surround inhibition is generated. One possible candidate is short intracortical inhibition (SICI). With SICI, a motor-evoked potential (MEP) produced by a suprathreshold stimulus is reduced in amplitude by a preceding subthreshold stimulus (Kujirai et al., 1993). SICI is $\mathrm{GABA}_{\mathrm{A}}$ mediated and thought to reflect the inhibitory effect of the horizontal interneurons in primary motor cortex (M1) (Kujirai et al., 
Table 1. Patient demographics

\begin{tabular}{|c|c|c|c|c|c|}
\hline Sex & Age (in years) & Type & Affected side & Duration (in years) & Botulinum toxin/last injection \\
\hline M & 44 & WC & Right & 25 & No \\
\hline M & 72 & WC & Right & 10 & Yes/4 months \\
\hline M & 56 & $M C$ & Right & 21 & No \\
\hline M & 48 & WC & Right & 6 & No \\
\hline M & 62 & WC & Bilateral & 39 & No \\
\hline M & 58 & WC & Right & 5 & Yes/3 months \\
\hline M & 55 & WC/MC & Right & 5 & Yes/4 months \\
\hline M & 60 & WC & Right & 26 & Yes/3 months \\
\hline M & 43 & WC & Bilateral & 23 & Yes/6 months \\
\hline $\mathrm{F}$ & 51 & WC & Right & 10 & Yes/3 months \\
\hline M & 57 & $M C$ & Right & 17 & Yes/3 months \\
\hline$F$ & 45 & WC & Bilateral & 23 & Yes/3 years \\
\hline M & 54 & $M C$ & Right & 19 & No \\
\hline$F$ & 47 & WC & Right & 4 & Yes/3 months \\
\hline M & 55 & $M C$ & Right & 3 & Yes/5 months \\
\hline M & 57 & $M C$ & Right & 5 & Yes/6 months \\
\hline
\end{tabular}

M, Male; F, female; WC, Writer's cramp; MC, musician's cramp

1993; Hanajima et al., 2003). SICI is reduced in the activated muscle during activation (Reynolds and Ashby, 1999; Stinear and Byblow, 2003), whereas it is increased contralaterally. Animal models also provide evidence of the crucial role that local GABAergic circuits have on motor output (Schneider et al., 2002). These observations support the concept that selectivity of a motor task is achieved by increasing the intracortical inhibition of the area surrounding the cortical representation of muscles acting as agonist or synergist in the particular task. Furthermore, impaired inhibitory circuits on the motor cortical level may be involved in the pathophysiology of dystonia (Hallett, 2004; Sohn and Hallett, 2004a; Stinear and Byblow, 2005).

The role of SICI in surround inhibition remains unclear and the results of two previous studies were contradictory (Stinear and Byblow, 2003; Sohn and Hallett, 2004b). The goal of the current study was to characterize further the generation of surround inhibition by assessing the modulation of SICI during the time course of a movement and by the comparison of healthy volunteers to a group of FHD patients, who are known to have reduced surround inhibition (Stinear and Byblow, 2004). Therefore, single- and paired-pulse TMS was applied at rest, before EMG onset in the active muscle, during movement initiation and during the maintenance of a contraction. We hypothesized that in healthy volunteers, surround inhibition would occur during movement initiation, but not during the maintenance phase, and it would be reduced in patients with FHD. This idea is derived from the clinical observation of FHD patients, in whom symptoms often start when initiating the movement, indicating the pivotal role of surround inhibition during movement initiation, although dystonic symptoms then result in sustained cocontractions. The current study aimed to determine for which movement phase surround inhibition is relevant. Furthermore, we assessed SICI reflecting intra-motor-cortical inhibition (Kujirai et al., 1993) to attain more insight in the generation of surround inhibition. We expected an enhancement of SICI during movement initiation in the control group (Stinear and Byblow, 2004), which would be absent in FHD patients.

\section{Materials and Methods}

Subjects. Sixteen FHD patients (age, 43-72 years; mean, $54.0 \pm 1.9$ years; 13 males) and 20 age-matched healthy subjects (age, 37-72 years; mean, $54.4 \pm 2.3$ years; 12 males) participated in the TMS part of the study. In six young healthy male volunteers (age, $24-33$ years; mean, $27.7 \pm 1.4$ years, all males), the Hoffmann reflex (H-reflex) in the abductor pollicis brevis muscle (APB) was tested. The incidence of the APB H-reflex is low and 32 people were screened overall to obtain the six that had H-reflexes in this muscle. Because the likelihood to evoke H-reflexes in any muscle decreases with age, we did not test the APB H-reflex in the subjects in the main experiment. All participants gave their informed consent before the experiments, which were approved by the Institutional Review Board of the National Institute of Neurological Disorders and Stroke. All subjects were right-handed according to the Edinburgh Handedness Inventory (Oldfield, 1971). Participants had never been treated with neuroleptic drugs and had no history of other neuropsychiatric disorders, neurosurgery, or metal or electronic implants. Most of the patients had been treated with local injections of botulinum toxin type $\mathrm{A}$ in the affected muscles. The last injection had been given at least 3 months before testing (Table 1).

Recording. Subjects were seated in a comfortable chair with their arm resting on a side table, which was individually adjusted. In some subjects, the wrist was supported by a towel to help the subject keep the hand muscles as relaxed as possible. Disposable surface silver-silver chloride EMG electrodes were placed on the right APB and first dorsal interosseus muscle (FDI) in a bipolar montage. Impedance was reduced to $<5 \mathrm{k} \Omega$. The EMG signal was amplified using a conventional EMG machine (Nihon Kohden) and bandpass filtered $(20-2000 \mathrm{~Hz})$. The signal was digitized at a frequency of $4 \mathrm{kHz}$ and fed into a computer for off-line analysis. The individual MEPs were measured in four phases (rest, premotor, phasic, and tonic) (see below, Motor task). The background EMG was calculated by assessing the root mean square $>50 \mathrm{~ms}$ before MEP onset in the same four phases (rest, premotor, phasic, and tonic).

Motor task. All tests were performed at rest or during different phases of an active movement of the FDI, which subjects practiced at the beginning of the experiment to attain a consistent motor performance. With their right hand lying flat on a table beside them, subjects pushed down on a small force transducer (Strain Measurement Devices; model S215 load cell) (see Fig. 1A) with the tip of their index finger in response to an acoustic signal. This led to a flexion in the metacarpo-phalangeal joint of the index finger. In preliminary tests, even healthy volunteers were not able to completely suppress EMG activity in thumb muscles, when the FDI was abducted (primary movement), because the thumb is then the only finger to oppose the movement under this condition to stabilize the hand. To minimize concomitant EMG activity in the APB, index-finger flexion was used. The FDI participates as a synergist rather than as prime mover in this motion, but it has been shown that the modulation of cortical excitability is similar in prime movers and synergists (Sohn and Hallett, 2004b).

Subjects were instructed to produce $10 \%$ of their maximum force right after the tone started, and to hold the contraction at the target force level of $10 \% F_{\max }$ for $2 \mathrm{~s}$. The force level was individually adjusted and dis- 
played as a line on an oscilloscope in front of them. The output of the force transducer was also displayed on the oscilloscope as feedback.

Four different phases of the contraction were assessed: rest $(100 \mathrm{~ms}$ before the onset of the tone), premotor (20 ms before the onset of the EMG in the FDI), phasic (the first peak of EMG in the FDI), and tonic (1600 ms after the onset of the acoustic signal, during contraction at the target force level of $10 \% F_{\max }$, maintenance phase) (see Fig. $1 B$ ). Whereas premotor and phasic phase are considered movement initiation phases, the tonic phase is regarded as maintenance of the contraction.

TMS. For TMS, two high-power Magstim $200^{2}$ machines, which have an integrated Bistim module, were connected to a Second Generation figure-of-eight coil (Remote 3190-00) with an inner-loop diameter of 70 $\mathrm{mm}$. The coil was placed over the "motor hot spot" for eliciting MEPs in the APB. This position was marked on the scalp to ensure proper coil placement through the experiment. Coil orientation was tangential to the scalp with the handle pointing backwards and laterally at a $45^{\circ}$ angle away from the midline, inducing a posterior-directed current in the brain to activate the corticospinal system, preferentially trans-synaptically (Di Lazzaro et al. 2004). The resting motor threshold (MT) was determined to the nearest $1 \%$ of maximal stimulator output. MT was defined as the minimal stimulus intensity required to evoke MEPs of at least $50 \mu \mathrm{V}$ in 5 of 10 consecutive trials. MEP size was determined by averaging peak-to-peak amplitudes. Trials with a background EMG of $>0.02 \mathrm{mV}$ in the APB (assessed as root mean square) over $50 \mathrm{~ms}$ before the onset of the MEP were rejected.

In the single-pulse TMS experiment, a stimulus intensity of $140 \%$ MT was used. Ten to 12 stimuli at four different, individually adjusted latencies (rest, premotor, phasic, and tonic) were applied in a randomized order with a fifth condition, where no TMS was given.

For SICI, an interstimulus interval of $2.5 \mathrm{~ms}$ was used, which has been shown to be most effective (Stinear and Byblow, 2004). The four different phases were tested in separate experiments. Test-pulse intensity was adjusted in the beginning of each experiment to induce a MEP of $1 \mathrm{mV}$. SICI was first performed at rest and the intensity of the conditioning stimulus was adjusted to reduce the size of the test pulse to $\sim 70 \%$, i.e., induce an SICI of 30\% (SICI $=[($ MEP test - MEP conditioned $) / \mathrm{MEP}$ test $] \times 100)$. This adjustment was performed to give room for an upregulation of SICI and to avoid saturation or floor effects (Fisher et al., 2002). This intensity for the conditioning pulse was then used for all other phases (Stinear and Byblow, 2004).

Electrical stimulation. The H-reflex is considered to be the electrical analog of the monosynaptic stretch reflex and to reflect spinal excitability while circumventing the muscle spindles (for review, see Zehr, 2002). For APB H-reflex stimulation, an electrical stimulator (Digitimer DS7) was used to generate single square-wave pulses of $1 \mathrm{~ms}$ duration. The bipolar stimulating electrode was placed over the median nerve at the wrist. APB H-reflex recruitment curves including the maximal amplitude of the $M$ response $\left(M_{\max }\right)$ were recorded at rest and during the same phases as described before (rest, premotor, phasic, and tonic). For each subject and phase, $M_{\max }$ and the maximal amplitude of H-reflex $\left(H_{\max }\right)$ were determined, and the $H_{\max } / M_{\max }$ ratio was calculated (Zehr, 2002).

Statistics. Outcome measures (peak-to-peak MEP amplitudes) were compared using a two-group repeated-measures ANOVA to compare the effect of "phase" as a within-subject factor (four levels: rest, premotor, phasic, and tonic) and "group" as a between-subject variable (two levels: FHD patients and controls). For the comparison of the background EMG, the within-subject factor "muscle" was also used (two levels: APB and FDI). Bonferroni's adjustment was used to correct for multiple comparisons. For the comparison of MT, an unpaired $t$ test was used. Data are presented as mean \pm SEM. $p$ values $<0.05$ are considered as significant. For analysis, SPSS 11.5 .0 was used.

\section{Results}

\section{Background EMG}

There were significant main effects for muscle $(F=29.4, p<$ $0.001)$ and phase $(F=15.5, p<0.001)$, but not for group $(p>$ $0.1)$. Figure $1 C$ illustrates that background EMG was modulated differently in the APB and FDI (muscle $\times$ phase interaction, $F=$ $15.5, p<0.001)$. Although background EMG was not different
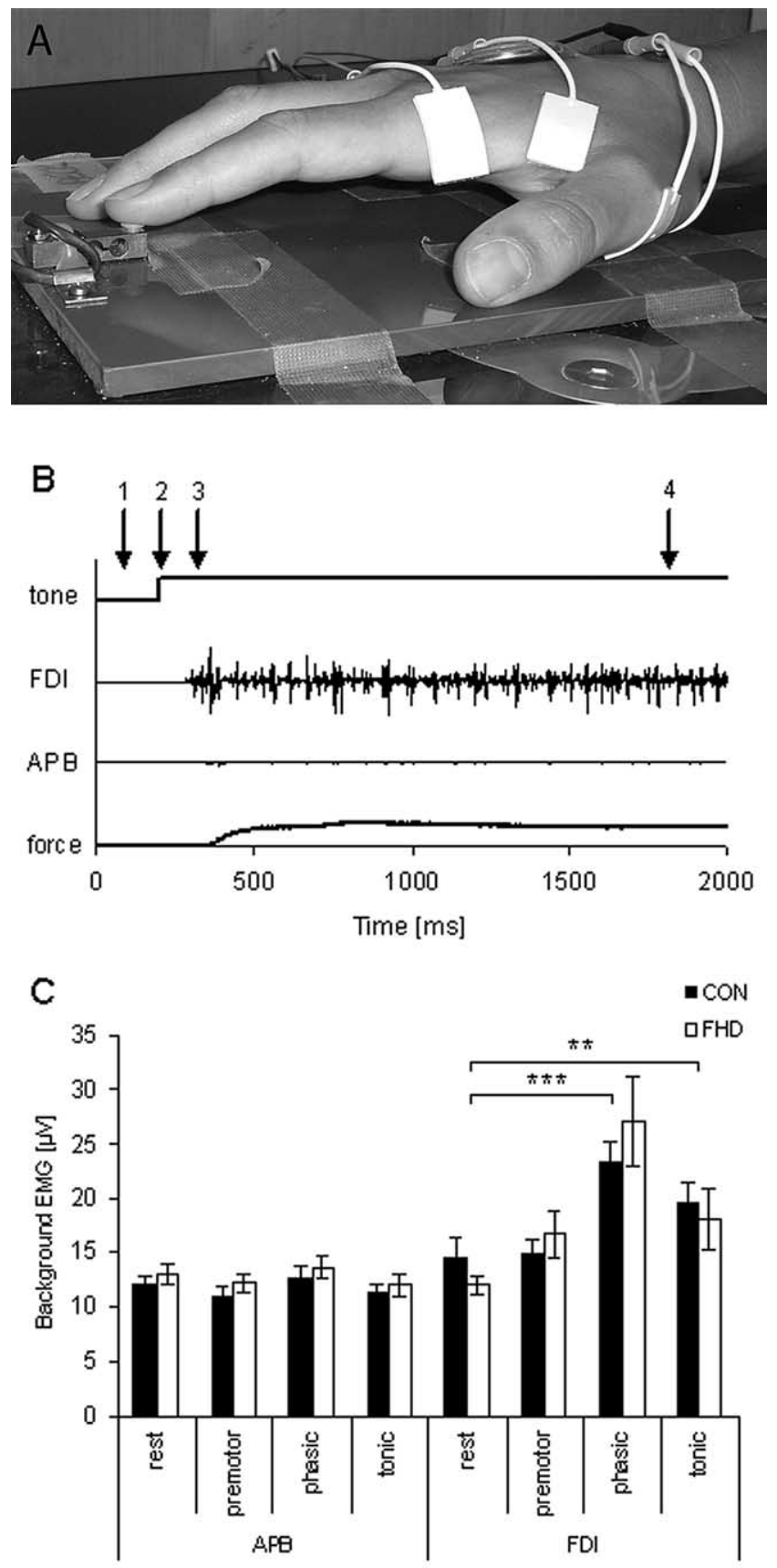

Figure 1. $\quad \boldsymbol{A}$, Experimental setup. Two pairs of surface electrodes were placed over the FDI and APB of the right hand, which is shown resting on a board. The tip of the index finger is resting on the force transducer. $\boldsymbol{B}$, Time course of the task. Shown are the acoustic signal, which starts $200 \mathrm{~ms}$ after the begin of the recording, EMG in the FDI, the synergistic muscle, EMG in the APB, the surrounding muscle, and the forced produced. Stimuli were applied before the onset of the tone (rest, after 100 $\mathrm{ms}$ ), during the premotor phase ( 20 ms before the onset of EMG in the FDI), the phasic phase (at the first peak of EMG in the FDI), and the tonic phase (1600 ms after the onset of the acoustic signal, maintenance phase of the contraction, steady state). C, Background EMG. Shown are the background EMG levels for the APB and FDI in all four different phases. Although there is no significant modulation of background EMG in APB, the EMG signal increases for the phasic and tonic phases in the FDI, reflecting its activation during the task. ${ }^{* *} p<0.01 ;{ }^{* * *} p<0.005$.

between phases in the APB (Table 2) (mean, $\sim 12 \pm 1 \mu \mathrm{V}$ ), it was increased for the phasic $(25 \pm 0.2 \mu \mathrm{V} ; F=41, p<0.001)$ and tonic phases $(19 \pm 0.2 \mu \mathrm{V} ; F=24.1, p<0.001)$ in the FDI, underlining its role as a synergist in the index-finger flexion. There were no other interactions (all $p>0.1$ ). 
Table 2. Single- and paired-pulse TMS in APB for all four phases of the movement

\begin{tabular}{|c|c|c|c|c|c|c|c|c|}
\hline & \multicolumn{4}{|l|}{$\mathrm{CON}$} & \multicolumn{4}{|l|}{ FHD } \\
\hline & Rest & Premotor & Phasic & Tonic & Rest & Premotor & Phasic & Tonic \\
\hline Single pulse (MEP size in mV) & $3.5 \pm 0.4$ & $3.0 \pm 0.4^{*, * *}$ & $2.3 \pm 0.4^{*, * *}$ & $4.3 \pm 0.6^{*}$ & $3.3 \pm 0.6$ & $3.3 \pm 0.6^{*}$ & $3.1 \pm 0.6^{*}$ & $3.6 \pm 0.7^{*}$ \\
\hline SICl test (MEP size in mV) & $1.2 \pm 0.1$ & $1.2 \pm 0.1$ & $1.2 \pm 0.1$ & $1.1 \pm 0.1$ & $1.4 \pm 0.2$ & $1.3 \pm 0.2$ & $1.1 \pm 0.1$ & $1.0 \pm 0.1$ \\
\hline SICI (\% test MEP) & $40.1 \pm 4$ & $33.9 \pm 6^{*}$ & $28.5 \pm 4^{*}$ & $28.9 \pm 7^{*}$ & $35.2 \pm 5$ & $21.1 \pm 9^{*, * *}$ & $3.6 \pm 8^{*, * *}$ & $22.1 \pm 8^{*}$ \\
\hline $\operatorname{EMG~APB~}(\mu \mathrm{V})$ & $12 \pm 0.9$ & $10.9 \pm 0.9$ & $12.7 \pm 0.9$ & $11.2 \pm 0.9$ & $13.1 \pm 1$ & $12.2 \pm 1$ & $13.7 \pm 1$ & $12 \pm 1.1$ \\
\hline
\end{tabular}

Shown are mean values and SEMs for single-pulse MEP size, test-pulse MEP size for the SICl experiments, SICl, and the background EMG in APB for both groups (FHD, $n=16 ;$ controls, $n=20$ ). CON, Control.

${ }^{*} p<0.05$, significant differences for this phase in both groups compared with the rest condition; ${ }^{* *} p<0.5$, significant differences for the group $\times$ phase interaction compared with the rest condition.

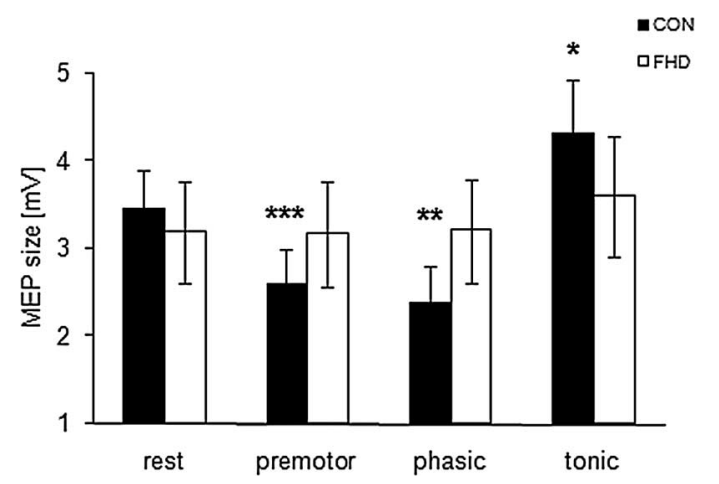

Figure 2. MEP size in APB. Shown are the mean MEP sizes with SEs in APB during the FDI movement for both groups (FHD patients and controls) during the four phases. Whereas the MEP size shows a clear inhibition just before and during the first phase of EMG onset in the adjacent muscle (FDI), there is an enhancement during the tonic contraction. Both modulations are not observable in the FHD patient group. ${ }^{*} p<0.05$; ${ }^{* *} p<0.01$; ${ }^{* * *} p<0.005$.

\section{Single-pulse TMS experiment}

In the control group, the pattern of modulation of MEP size in the APB for the different phases showed clear inhibition during the initiation of the index-finger flexion and enhancement during the tonic phase of the contraction (for means and SEs, see Table 2). Although the main effect for group was not significant ( $p=0.88)$, a significant main effect was found for phase $(F=7.9$, $p<0.001)$, and the group $\times$ phase interaction was also significant $(F=4.7, p=0.009)$ (Fig. 2). Simple contrasts were used to test for differences between rest and the three active levels of the factor phase. All phases differed from rest (premotor, $F=14.6$, $p=0.001$; phasic, $F=9.4, p=0.004$; tonic, $F=9.7, p=0.004)$. For the group $\times$ phase interaction, only the premotor $(F=14.6$, $p=0.001)$ and phasic $(F=9.4, p=0.004)$ phases showed a reduction in MEP size compared with rest, indicating a differential inhibition of the MEP in the APB in the control group during both movement initiation phases. The FHD patients did not show such inhibition (all $p>0.1$ ). The facilitating effect on the MEP in the tonic phase did not differ between patients and controls $(p>0.1)$.

When analyzing the groups separately, there was no significant modulation of APB MEP size by phase in the FHD patients $(F=0.9, p=0.46)$. In contrast, there was significant modulation in the control group $(F=9.4, p=0.001)$. For the factor phase, all active levels were different from rest in the control group (premotor, $F=24.3, p<0.001$; phasic, $F=15.6, p=0.001$; tonic, $F=8.3, p=0.01$ ). Whereas premotor and phasic phase were inhibited, the tonic phase was facilitated.

MT for the APB did not differ between FHD patients (44.7 \pm $2.4 \%)$ and the control group $(47.5 \pm 2.0 \% ; F=0.8, p=0.39)$.

In the FDI, MEP size was also different between the four phases $(F=12.6, p<0.001)$ and increased for all active phases as reflected by the contrasts (premotor, $F=11.7, p=0.02$; phasic,

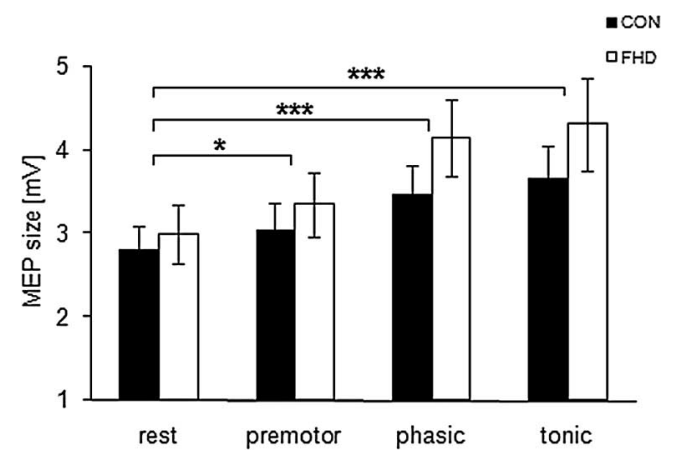

Figure 3. MEP size in FDI. Shown are the mean MEP sizes with SEs in FDI during movement for both groups (FHD patients and controls) during the four phases. MEP size shows an increase for all active tasks compared with rest underlining its active role in the selected movement. There was no difference in modulation between FHD patients and controls. ${ }^{*} p<0.05$; ${ }^{* *} p<$ $0.01 ; * * * 0.005$.

$F=33.1, p<0.001$; tonic, $F=31.0, p<0.001$ ) (Fig. 3). This agrees with the findings for the background EMG and underlines the role of FDI as synergist in the motor task. There was no difference between groups $(p>0.1)$.

\section{Paired-pulse TMS experiment}

SICI was calculated, and is shown as the percentage reduction of the MEP size with reference to the test MEP. The target reduction was 30\% for the rest condition. In fact, the mean of the induced SICI was a little higher, but it was not different between groups [SICI (rest), $35.2 \pm 5 \%$ in the FHD patient group and $40.1 \pm 4 \%$ in the control group; $F=0.7, p=0.40$ )] (Fig. 4).

There was a significant main effect for phase $(F=9.4, p<$ $0.001)$, for group $(F=10.6, p=0.003)$, and for the group $\times$ phase interaction $(F=8.1, p<0.001)$. Again, simple contrasts were used to test for differences between each of the three active levels of the factor phase and the first level (rest) as the reference category. SICI was smaller for the premotor, phasic, and tonic phases compared with rest (premotor SICI, $28 \pm 4 \%, F=10.4$, $p=0.003$; phasic SICI, $19.1 \pm 4 \%, F=27.8, p<0.001$; tonic SICI, $26.1 \pm 5 \%, F=6.2, p=0.017)$. For the group $\times$ phase interaction, contrasts showed that SICI in the premotor $(F=7.3$, $p=0.01)$ and phasic phase $(F=16.6, p<0.001)$ was reduced in the FHD patients (for means and SEs, see Table 2).

When analyzing the FHD group separately, there was a significant modulation of SICI by phase $(F=10, p=0.001)$. Calculation of simple contrasts indicated highly significant reduction of SICI for the premotor $(F=13.5, p=0.003)$ and phasic $(F=33$, $p<0.001)$ phases, although there was a trend for a decrease in SICI for the tonic phase (tonic, $F=4.5, p=0.05$ ). In the control group, there was no modulation of SICI during the different phases (all $p>0.1)$.

For the test MEP size, there were no significant main effects of 


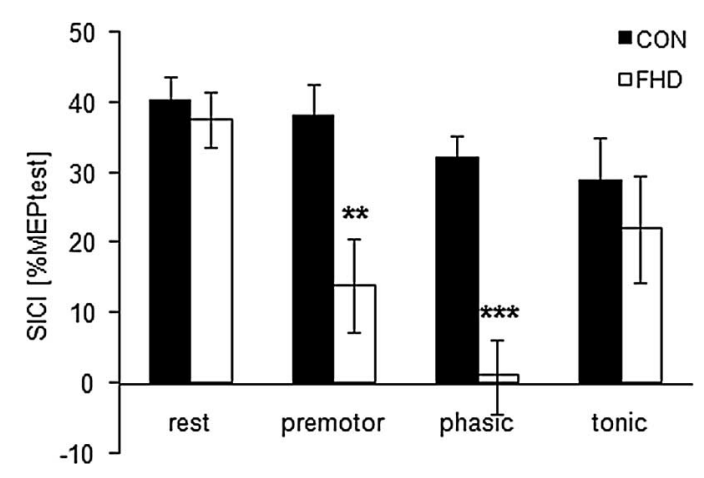

Figure 4. $S I C l$ is shown as group mean percentage change $[S I C l=(M E P$ test $-M E P$ conditioned/MEP test) $\times 100$ ] with SEs. For the rest condition and tonic state, there is no difference between FHD patients and controls. For patients, $\mathrm{SICl}$ is reduced during premotor and phasic phases of the adjacent FDI contraction. In the control group, $\mathrm{SICl}$ shows no phase-specific modulation. ${ }^{* *} p<0.01 ;{ }^{* *} p<0.005$.

group, phase, or the Group $\times$ phase interaction (all $p>0.1$ ) (for means and SEs, see Table 2).

\begin{abstract}
APB H-reflex
As a control experiment for possible contributions of changes in spinal excitability, we also tested the H-reflex in the APB during the four phases of movement in the FDI in a separate group of healthy subjects. The results showed a significant main effect for phase $(F=3.2, p=0.049)$. Using simple contrasts to test for differences between the three active levels for the factor phase and rest $(H / M$ rest, $4.5 \pm 1.4)$ as the reference category, we observed an increased $H_{\max } / M_{\max }$ ratio for the phasic phase $(H / M=6.5 \pm$ $1.6, F=9.3, p=0.03)$. There was a trend for an increased $H_{\max } l$ $M_{\max }$ ratio for the premotor phase $(H / M=6.4 \pm 2.0, F=4.6$, $p=0.08)$, although the $H_{\max } / M_{\max }$ ratio did not differ significantly for the tonic phase $(H / M=4.0 \pm 0.8, F=1.3, p=0.56)$ (Fig. 5).
\end{abstract}

\section{Discussion}

The results from the healthy subjects further support the hypothesis that surround inhibition, as assessed by an inhibition of MEP size, occurs in the inactive APB during index-finger flexion. Furthermore, surround inhibition seems to be sculpted in time and space. Although the inhibition of noninvolved muscles has only been reported between hand muscles so far (Sohn and Hallett, 2004a; Stinear and Byblow, 2004), the current results show that it is restricted to the movement initiation phase and absent during tonic contraction. Surround inhibition may represent a cortical mechanism to counteract increased spinal excitability during movement initiation while preserving motor precision. This brief increase in spinal excitability is the Jendrássik effect and is not spatially selective (Zehr and Stein 1999). The role for a generalized, phasic increase in spinal excitability with movement is not clear, but may be helpful in rapid movement initiation because spinal $\alpha$-motoneurons in the rest state are far from threshold (unlike many neurons in the nervous system, they do not show spontaneous activity). During the subsequent maintenance phase, spinal excitability decreased to resting levels, indicating that in this phase of the movement, surround inhibition may no longer be necessary. Movement selection would then have to arise from the excitatory command itself, which is well known not to be very selective or limited to the neurons projecting on the target muscle as might be expected (Slobounov et al., 2002; Schieber, 2004).

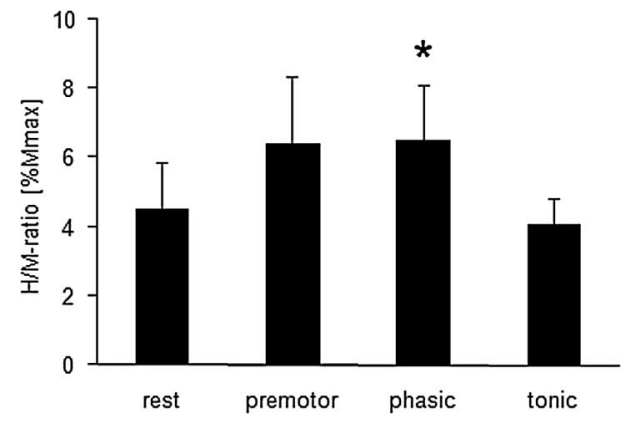

Figure 5. The graph shows mean and SE of the $H_{\max } / M_{\max }$ ratios assessed in six young healthy volunteers. The $H_{\max } / M_{\max }$ ratio is increased during the initiation phase of FDI contraction and returns to baseline during the tonic contraction phase. ${ }^{*} p<0.05$.

Whereas healthy volunteers showed a reduction of MEP size in the quiescent APB during the first phase of a contraction of the FDI, patients with FHD did not modulate APB MEP size during movement initiation. Using a paired-pulse TMS paradigm to assess the contribution of the intracortical inhibition, it was shown that this lack of modulation of the APB MEP size was accompanied by reduced SICI in FHD patients during movement initiation. Therefore, SICI may contribute to the inhibitory network shaping surround inhibition. Thus, reduced SICI appears to play a role in impaired surround inhibition in FHD.

For the maintenance phase, there was no surround inhibition observable in either group, suggesting that phasic and tonic phases use different mechanisms. In fact, APB MEP size was enhanced overall and, significantly, in the control group during tonic contraction. SICI was unaltered in both groups in this phase, suggesting that this inhibitory cortical circuit is not involved in modulating MEP size during this phase.

To assess changes in spinal excitability, the APB H-reflex was monitored during the same four phases. The $H / M$-ratio showed increased spinal excitability during movement initiation (premotor and phasic phase), but not during the maintenance phase. Hence, there was no spinal inhibition, but a facilitation, which shows that the inhibition is most likely supraspinal, and the spinal contribution to both phenomena, surround inhibition and MEP facilitation during tonic contraction, is probably negligible (Sohn and Hallett, 2004a).

A typical clinical feature of FHD is a task- and context-specific involuntary spread of muscle contractions (Chen and Hallett, 1998). Abnormal movements commonly occur during movement initiation and then result in long-lasting, tonic contractions of antagonistic and other nearby muscles (Cohen and Hallett, 1988). There is evidence that surround inhibition could help the selective execution of desired movements in humans (Hallett, 2004; Sohn and Hallett, 2004b). The horizontal network of local intracortical interneurons plays an important role in shaping the output from M1 (Donoghue and Sanes, 1994). In animal studies, it has been shown that the application of $\mathrm{GABA}_{\mathrm{A}}$-antagonist drugs leads to a spread of cortical excitability (Schneider et al., 2000). In addition, $\mathrm{GABA}_{\mathrm{A}}$-agonist drugs increase SICI in humans (Ziemann, 2004) and are being used as a treatment for dystonia. In FHD, increased motor cortical excitability (Ikoma et al., 1996) and deficient intracortical inhibitory circuits have been demonstrated in active (Chen et al., 1995, 1997; Gilio et al., 2000; Rona et al., 1998) as well as in adjacent relaxed muscles (Hallett, 2004; Sohn and Hallett, 2004a; Stinear and Byblow, 2005).

In two previous studies (Sohn and Hallett, 2004a; Stinear and Byblow, 2004), the role of SICI in surround inhibition has been 
examined with contradictory results. One study used a selfinitiated index-finger flexion and had the abductor digiti minimi muscle $(\mathrm{ADM})$ as the target muscle. Although there was clear surround inhibition, as reflected by decreased MEP size, SICI was not modulated (Sohn and Hallett, 2004a). In a second study, a rhythmic index-finger flexion of $1 \mathrm{~Hz}$ was used and the FDI and APB were compared. In this study, the control group showed enhanced SICI in the APB during movement initiation in the FDI (Stinear and Byblow, 2004). Furthermore, and very much in line with the data presented here, SICI was reduced in FHD patients, although this effect did not reach statistical significance in the seven patients tested in this study.

Several reasons may account for this discrepancy in SICI test results. First, it is known that cortical inhibition in general tends to decrease with age (Peinemann et al., 2001; Hortobágyi et al., 2006; Rossini et al., 2007). In addition, reduced capacity to upregulate SICI during a self-initiated movement compared with rest may be attributable to a saturation effect, also known as "floor effect" (Fisher et al., 2002), which might be even be more important in elderly people, in whom SICI is already reduced (Peinemann et al., 2001; Hortobágyi et al., 2006). Therefore, the intensity of the conditioning stimulus and the baseline inhibition seem to be crucial. Whereas the previous study by Stinear et al. (2004) reported a significant increase in SICI in the control group starting from a very low level of SICI $(25.8 \%)$, the abovementioned study by Sohn and Hallett (2004b) was unable to reproduce this using a baseline SICI of $\sim 60 \%$ (Sohn and Hallett, 2004a). In the current study, SICI at rest ended up to be at a higher level $(40.1 \pm 4.1 \%)$ than initially aimed for $(30 \%)$. This may explain the different results.

Second, the task itself may account for the differences. Whereas Sohn et al. (2004) used a very slight, self-triggered movement, the rhythmic task assessed in the experiment by Stinear et al. (2004), as well as the current task, involved an external acoustic trigger, and feedback of the performance was given directly, either acoustically or visually. Both latter tasks required feedback control with a high precision of motor execution. Subjects had to adapt their movement either to a strict, fast rhythm (Stinear and Byblow, 2004) or, in the present task, to a precise force level designed as an acoustic reaction time task.

Third, although surround inhibition during index-finger flexion affected thumb and fifth finger muscles (Sohn and Hallett, 2004a; Stinear and Byblow, 2004), the amount of surround inhibition may depend on the degree to which the muscles are actually unrelated. For instance, index finger and fifth finger (e.g., during gripping or reaching), may have a smaller amount of surround inhibition compared with muscles that are more commonly used independently, such as the index finger and thumb muscles. Additionally, intracortical inhibitory networks differ between intrinsic hand muscles and generally are stronger in the FDI than in ADM (Takahashi et al., 2005).

Another issue that may explain the discrepancy between the literature and our results is statistical power. The first study showed also a decrease of SICI in the patient group during phasic contraction (Stinear and Byblow, 2004), which did not reach statistical significance in the seven patients tested. With a higher number of FHD patients $(n=16)$, we detected this reduction of SICI, which is in accordance with the previous trend.

The current results are in line with a previous abstract of deficient SICI in surrounding muscles just before EMG onset (Molloy et al., 2002), indicating that surround inhibition is relevant during the movement initiation phase. In contrast, facilitation of MEP size was observed during the maintenance phase (tonic).
Because there was no enhancement of spinal excitability in this phase, the modulation most likely occurs on a supraspinal level. The enhancement in MEP size reached statistical significance only for the control group. In this regard, it needs to be mentioned that none of the patients had dystonic posturing during the test. The absent MEP facilitation in FHD patients, as opposed to controls, may either be attributable to low power or indicate a compensatory effect by cortical inhibitory circuits other than SICI, which remained unchanged during this phase in both groups. Because it has been shown that SICI can be increased voluntarily (Sohn et al., 2002), we cannot completely rule out that a voluntary increase of SICI in the patient group may be masking a potential deficit in this circuit. Possible candidates for a compensatory increase in inhibition of M1 would be inhibitory interhemispheric projections (Ferbert et al., 1992), although they are known to project onto the local interneurons mediating SICI (Chen, 2004).

In conclusion, the current study extends the previous findings by showing that, on the motor cortical level, surround inhibition is sculpted in time and space, being primarily relevant during movement initiation and not relevant during tonic contraction. Thus, surround inhibition may have an important role for individuation of phasic, fine finger movements. The current results confirm impaired surround inhibition in FHD patients during movement initiation and establish, at least in part, an association with a reduction of SICI during phasic contraction. This decreased intracortical inhibition may lead to reduced contrast on the motor cortical level and thereby hamper fast manual tasks and contribute to the overflow of muscle activation seen in dystonic movement.

\section{References}

Angelucci A, Levitt JB, Lund JS (2002) Anatomical origins of the classical receptive field and modulatory surround field of single neurons in macaque visual cortical area V1. Prog Brain Res 136:373-388.

Blakemore C, Carpenter RH, Georgeson MA (1970) Lateral inhibition between orientation detectors in the human visual system. Nature 228:37-39.

Chen R (2004) Interactions between inhibitory and excitatory circuits in the human motor cortex. Exp Brain Res 154:1-10.

Chen R, Hallett M (1998) Focal dystonia and repetitive motion disorders. Clin Orthop Relat Res 102-106.

Chen R, Wassermann EM, Caños M, Hallett M (1997) Impaired inhibition in writer's cramp during voluntary muscle activation. Neurology 49:1054-1059.

Chen R, Tam A, Bütefisch C, Corwell B, Ziemann U, Rothwell JC, Cohen LG (1998) Intracortical inhibition and facilitation in different representations of the human motor cortex. J Neurophysiol 80:2870-2881.

Chen RS, Tsai CH, Lu CS (1995) Reciprocal inhibition in writer's cramp. Mov Disord 10:556-561.

Cohen LG, Hallett J (1988) Hand cramps: clinical features and electromyographic patterns in a focal dystonia. Neurology 38:1105-1012.

Di Lazzaro V, Oliviero A, Pilato F, Saturno E, Dileone M, Mazzone P, Insola A, Tonali PA, Rothwell JC (2004) The physiological basis of transcranial motor cortex stimulation in conscious humans. Clin Neurophysiol 115:255-266.

Denny-Brown D (1967) The fundamental organization of motor behavior. In: Neurophysiological basis of normal and abnormal motor activities (Yahr M, Purpura D, eds), pp 415-442. New York: Raven.

Donoghue JP, Sanes JN (1994) Motor areas of the cerebral cortex. J Clin Neurophysiol 11:382-396.

Doumas M, Wing AM, Wood K (2008) Interval timing and trajectory in unequal amplitude movements. Exp Brain Res 189:49-60.

Ferbert A, Priori A, Rothwell JC, Day BL, Colebatch JG, Marsden CD (1992) Interhemispheric inhibition of the human motor cortex. J Physiol 453:525-546.

Fisher RJ, Nakamura Y, Bestmann S, Rothwell JC, Bostock H (2002) Two 
phases of intracortical inhibition revealed by transcranial magnetic threshold tracking. Exp Brain Res 143:240-248.

Gilio F, Currà A, Lorenzano C, Modugno N, Manfredi M, Berardelli A (2000) Effects of botulinum toxin type A on intracortical inhibition in patients with dystonia. Ann Neurol 48:20-26.

Hallett M (2003) Surround inhibition. Suppl Clin Neurophysiol $56: 153-159$.

Hallett M (2004) Dystonia: abnormal movements result from loss of inhibition. Adv Neurol 94:1-9.

Hanajima R, Furubayashi T, Iwata NK, Shiio Y, Okabe S, Kanazawa I, Ugawa Y (2003) Further evidence to support different mechanisms underlying intracortical inhibition of the motor cortex. Exp Brain Res 151:427-434.

Hortobágyi T, Del Olmo MF, Rothwell JC (2006) Age reduces cortical reciprocal inhibition in humans. Exp Brain Res 171:322-329.

Ikoma K, Samii A, Mercuri B, Wassermann EM, Hallett M (1996) Abnormal cortical motor excitability in dystonia. Neurology 46:1371-1376.

Kujirai T, CaramiaMD, Rothwell JC, Day BL, Thompson PD, Ferbert A, Wroe S, Asselman P, Marsden CD (1993) Corticocortical inhibition in human motor cortex. J Physiol 471:501-519.

Mink JW (1996) The basal ganglia: focused selection and inhibition of competing motor programs. Prog Neurobiol 50:381-425.

Molloy FM, Sohn YH, Hallett M (2002) Surround inhibition is impaired in patients with focal hand dystonia during movement preparation. Mov Disord 17 [Suppl 5]:304-305.

Oldfield RC (1971) The assessment and analysis of handedness: the Edinburgh inventory. Neuropsychologia 9:97-113.

Peinemann A, Lehner C, Conrad B, Siebner HR (2001) Age-related decrease in paired-pulse intracortical inhibition in the human primary motor cortex. Neurosci Lett 313:33-36.

Reynolds C, Ashby P (1999) Inhibition in the human motor cortex is reduced just before a voluntary contraction. Neurology 53:730-735.

Rona S, Berardelli A, Vacca L, Inghilleri M, Manfredi M (1998) Alterations of motor cortical inhibition in patients with dystonia. Mov Disord 13:118-124.

Rossini PM, Rossi S, Babiloni C, Polich J (2007) Clinical neurophysiology of aging brain: From normal aging to neurodegeneration. Prog Neurobiol 83:375-400.
Schieber MH (2004) Motor control: basic units of cortical output? Curr Biol 14:R353-R354.

Schneider C, Lavoie BA, Capaday C (2000) On the origin of the soleus H-reflex modulation pattern during human walking and its taskdependent differences. J Neurophysiol 83:2881-2890.

Schneider C, Devanne H, Lavoie BA, Capaday C (2002) Neural mechanisms involved in the functional linking of motor cortical points. Exp Brain Res 146:86-94.

Shin HW, Kang SY, Sohn YH (2007) Disturbed surround inhibition in preclinical parkinsonism. Clin Neurophysiol 118:2176-2179.

Slobounov S, Johnston J, Chiang H, Ray WJ (2002) Motor-related cortical potentials accompanying enslaving effect in single versus combination of fingers force production tasks. Clin Neurophysiol 113:1444-1453.

Sohn YH, Hallett M (2004a) Disturbed surround inhibition in focal hand dystonia. Ann Neurol 56:595-599.

Sohn YH, Hallett M (2004b) Surround inhibition in human motor system. Exp Brain Res 158:397-404.

Sohn YH, Wiltz K, Hallett M (2002) Effect of volitional inhibition on cortical inhibitory mechanisms. J Neurophysiol 88:333-338.

Stinear CM, Byblow WD (2003) Role of intracortical inhibition in selective hand muscle activation. J Neurophysiol 89:2014-2020.

Stinear CM, Byblow WD (2004) Impaired modulation of intracortical inhibition in focal hand dystonia. Cereb Cortex 14:555-561.

Stinear CM, Byblow WD (2005) Task-dependent modulation of silent period duration in focal hand dystonia. Mov Disord 20:1143-1151.

Takahashi M, Ni Z, Yamashita T, Liang N, Sugawara K, Yahagi S, Kasai T (2005) Differential modulations of intracortical neural circuits between two intrinsic hand muscles. Clin Neurophysiol 116:2757-2764.

Wing AM (2002) Voluntary timing and brain function: an information processing approach. Brain Cogn 48:7-30.

Zehr EP, Stein RB (1999) Interaction of the Jendrássik maneuver with segmental presynaptic inhibition. Exp Brain Res 124:474-480.

Zehr PE (2002) Considerations for use of the Hoffmann reflex in exercise studies. Eur J Appl Physiol 86:455-468.

Ziemann U (2004) TMS and drugs. Clin Neurophysiol 115:1717-1729.

Ziemann U, Rothwell JC, Ridding MC (1996) Interaction between intracortical inhibition and facilitation in human motor cortex. J Physiol 496: 873-881. 